\title{
The spectral scalings of magnetic fluctuations upstream and downstream of the Venusian bow shock
}

\author{
S. D. Xiao ${ }^{1}$, M. Y. Wu ${ }^{1}$, G. Q. Wang ${ }^{1}$, Y. Q. Chen ${ }^{2}$ and T. L. Zhang ${ }^{1,3^{*}}$
}

\begin{abstract}
We statistically investigate the spectral scalings of magnetic fluctuations at the upstream and downstream regions near the Venusian bow shock and perform a differentiation by shock geometry. Based on the Venus Express data, 115 quasi-parallel $\left(Q_{\|}\right)$bow shock crossings and 303 quasi-perpendicular $\left(Q_{\perp}\right)$ bow shock crossings are selected. The statistical results suggest that the bow shock tends to modify the upstream spectra flatter to $1 / f$ noise in the magnetohydrodynamics (MHD) regime and steeper to turbulence in the kinetic regime after the magnetic fluctuations crossing the bow shock, and this modification for the $Q_{\|}$and $Q_{\perp}$ bow shocks is basically consistent. However, the upstream spectral scalings are associated with the shock geometry. The changes of the spectral scalings of magnetic fluctuations near the $Q_{\|}$bow shocks are not as significant as near the $Q_{\perp}$ bow shock crossings. That might result from the fluctuations generated by the backstreaming ions which can escape across the $Q_{\|}$bow shock into the foreshock. Our results suggest that the energy cascade and dissipation near Venus can be modified by the Venusian bow shock, and the $Q_{\|}$bow shock plays an important role on the energy injection and dissipation in the solar wind interaction with Venus. The large dispersion of spectral scalings indicates that this fluctuation environment is complicated, and the shock geometry is not the only key factor in the fluctuations across the Venusian bow shock. Other possible factors in the shock modification to the upstream fluctuations will be explored in future.
\end{abstract}

Keywords: Magnetic fluctuations, Turbulence, Venusian bow shock, Spectral scalings, Shock geometry, Venus Express

\section{Introduction}

As a typical unmagnetized planet, Venus has no global intrinsic magnetic field. An induced magnetosphere is created by the solar wind (SW) interaction with Venus (e.g., Zhang et al. 2008a), consisting of the magnetic barrier (e.g., Zhang et al. 1991, 2008b; Xiao and Zhang 2018) and the magnetotail (e.g., Rong et al. 2014; Xiao et al. 2016). The Venusian-induced magnetosphere can deflect the upstream SW, and a bow shock and a magnetosheath are formed above (e.g., Zhang et al. 2008c; Phillips and McComas 1991). The near-Venusian space environment is a natural laboratory to investigate the SW interaction with unmagnetized planetary bodies (e.g., Luhmann

*Correspondence: Tielong.Zhang@oeaw.ac.at

${ }^{1}$ Harbin Institute of Technology, Shenzhen, China

Full list of author information is available at the end of the article
1986). In the near-Venusian space, magnetic field fluctuations play an important role in the transformation of momentum and energy, and their properties are widely reported (e.g., Luhmann et al. 1983; Guicking et al. 2010; Du et al. 2010; Xiao et al. 2017). The power of these fluctuations generally exhibits a frequency function as $P \propto 1 / f^{\alpha}$ (where $P$ is the power spectral density (PSD), $f$ is the frequency, and $\alpha$ is the spectral scaling index). The index $\alpha$ is considered as an indicator of the nature of fluctuations, and it generally has different values for the frequency ranges above and below the local proton gyrofrequency $\left(f_{p}=e q / m_{p}\right)$. In the magnetohydrodynamics (MHD) frequency range, the Kolmogorov scaling value of $\alpha \sim 5 / 3$ is expected for turbulence. Energy cascade occurs in this regime, where the energy is transferred from lower to higher frequencies. In the kinetic frequency range, the turbulence has a larger value of $\alpha \sim 2.8$, in which regime 
the magnetic energy dissipates into plasma or accumulates by exciting some dispersive waves, like the whistler. The spectral scaling indices have been extensively used to analyze the magnetic field fluctuations and turbulence in the solar wind (e.g., Alexandrova et al. 2008, 2009; Kiyani et al. 2009; Bruno and Carbone 2013), in the planetary space environments near Earth (e.g., Vörös et al. 2004, 2007; Vörös 2011), Mars (e.g., Ruhunusiri et al. 2017), and Venus (e.g., Vörös et al. 2008a, b; Dwivedi et al. 2015; Xiao et al. 2018, 2020a, b).

Although Venus has a similar size compared to Earth, the Venusian bow shock and magnetosheath are much smaller, with a scale ratio of $\sim 1 / 10$ (e.g., Slavin et al. 1979). This might result in some differences between their space plasma environments. Vörös et al. (2008a, b) reported a survey of the spectral scalings of magnetic fluctuations in the Venusian magnetosheath and wake. They observed $1 / f$ noise in the dayside magnetosheath, wavy structures near the terminator, and MHD turbulence at the magnetosheath post terminator boundary layer and near the nightside bow shock. The observed $1 / f$ noise in the Venusian magnetosheath may indicate that the energy cascade between different scales is absent and the fluctuations are controlled by multiple uncorrelated driving sources (e.g., Vörös et al. 2007). Xiao et al. (2018) further examined the magnetic fluctuations in the dayside Venusian magnetosheath and found a clear difference of the turbulence distributions between downstream of the quasi-parallel $\left(Q_{\|}\right)$and the quasiperpendicular $\left(Q_{\perp}\right)$ shocks. It is speculated that turbulence can be rapidly developed along the streamlines or penetrate into the Venusian magnetosheath downstream of the $Q_{\|}$bow shock. It suggests that the shock geometry has an effect on the spectral scalings of downstream magnetic fluctuations, and the bow shock plays a role on the turbulence distribution in the near-Venusian space. Xiao et al. (2020a) presented a description of the spectral scalings of magnetic fluctuations at the Venusian bow shock crossings. In terms of the spectral scalings, the dayside-nightside shock crossings exhibit a clear asymmetry. Noisy fluctuations dominate at the dayside shock crossing, and more MHD turbulence is present at the nightside shocks. Moreover, this distribution at the bow shock seems independent on the shock geometry. However, we still rarely know how the bow shock modifies the upstream magnetic fluctuations and turbulence from the SW.

Besides, previous investigations of turbulence near Venus are mainly focused on the MHD regime. It is believed that the spectral scalings of magnetic field fluctuations in the kinetic regime are quite different from those in the MHD regime. A recent research presented the global spatial distribution of the spectral scaling indices of magnetic fluctuations in both of MHD and kinetic regimes, and the global distribution suggests that the kinetic effects on magnetic energy dissipation are common in the near-Venusian space (Xiao et al. 2020b). The SW turbulence can be modified by the Venusian bow shock in both MHD and kinetic regime, and kinetic turbulence extensively occurs in the Venusian magnetosheath and the induced magnetosphere. It is believed that kinetic turbulence plays a prominent role in magnetic energy dissipation and particle heating in such an environment.

In this paper, we aim to differentiate the spectral scalings of the magnetic field fluctuations upstream and downstream of the Venusian bow shock and the shock geometry effects. The spectral scaling indices will be examined in both MHD and kinetic regimes. The investigation of the Venusian bow shock modifications to the upstream SW turbulence can help us better understand the energy injection, cascade, and dissipation in the SW interaction with Venus.

\section{Data and methods}

Venus Express (Svedhem et al. 2007; Titov et al. 2006), as the first ESA's Venus exploration mission, was launched in November 2005 and arrived at Venus in April 2006. Venus Express provides a great opportunity to examine the spectral scalings of the magnetic fluctuations near the Venusian bow shock. In this study, the magnetic field data measured by Venus Express magnetometer (Zhang et al. 2006) at a sampling rate of $32 \mathrm{~Hz}$ are used, and the bow shock crossings can be identified by the sudden change in the magnitude of the magnetic field between the SW and the Venusian magnetosheath, as shown in Fig. 1a.

Figure 1a shows the total magnetic field time series containing a bow shock crossing on Dec. 30, 2006. To examine the spectral scalings of the upstream and downstream magnetic fluctuations near the Venusian bow shock, the upstream and downstream intervals need to be selected during this shock crossing event. For this inbound case, a 256-s upstream interval is selected before the shock, and a 128-s downstream interval is selected after the shock crossing, as indicated in Fig. 1b. Figure 1b shows the magnetic field observations near the bow shock crossing in the aberrated Venus solar orbital (VSO) coordinate system, where the $X$ axis points antiparallel to the average $S W$ flow (with an aberration angle of $5^{\circ}$ ) from Venus, the $Z$ axis is perpendicular to the ecliptic plane and toward north, and the $Y$ axis completes the righthanded Cartesian coordinate system. Based on these two intervals, the PSDs are calculated via wavelet transforms (Torrence and Compo 1998): 


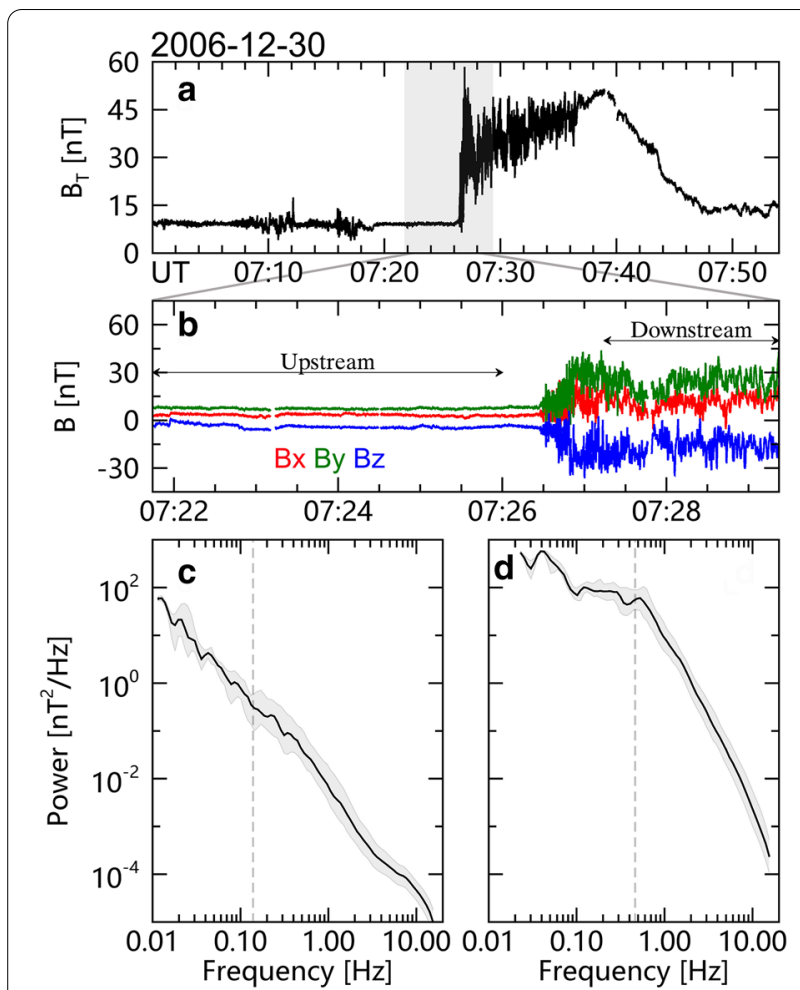

Fig. 1 A Venusian bow shock crossing event observed by Venus Express on 30 Dec. 2006. a The time series of the total magnetic field, and the shock crossing interval is shown in shadow. $\mathbf{b}$ The magnetic field observations near the bow shock in the aberrated VSO coordinate system, and the upstream and downstream intervals are indicated. $\mathbf{c}, \mathbf{d}$ The PSDs of the magnetic fluctuations for the upstream and downstream intervals

$$
\operatorname{PSD}(f)=\frac{2 \Delta t}{N} \sum_{j=1}^{N}\left[W_{x}^{2}\left(t_{j}, f\right)+W_{y}^{2}\left(t_{j}, f\right)+W_{z}^{2}\left(t_{j}, f\right)\right],
$$

where $\Delta t$ is the sampling time, $N$ is the length of the time series, and $W_{x}, W_{y}$, and $W_{z}$ are the wavelet transforms of the $x, y$, and $z$ components of the magnetic field, respectively.

The upstream and downstream PSDs are, respectively, shown in Fig. 1c, d with dashed $f_{p}$. An obvious spectral break near the $f_{p}$ is present in the downstream PSD, while it is not so clear for the upstream. The spectral break between the MHD and kinetic regimes is generally around $f_{p}$. However, the spectral break is not always precisely at $f_{p}$. For example, the spectral break may correlate better with the ion plasma frequency under some conditions (Chen et al. 2014). Due to the lack of ion data, here we can distinguish these two regimes using $f_{p}$ and ignore the transition range around $f_{p}$ to eliminate the interference. In this study, we refer to the frequency range below $f_{p} / 2$ as the MHD regime and the frequency range above $2 f_{p}$ as the kinetic regime. The value of $f_{p}$ is typically exhibited as $0.1 \mathrm{~Hz}$ in the upstream SW near Venus and $0.3 \mathrm{~Hz}$ in the downstream Venusian magnetosheath. The upstream interval is selected longer to cover a lower frequency range. Then the spectral indices in the MHD regime $\left(\alpha_{\mathrm{m}}\right)$ and the kinetic regime $\left(\alpha_{\mathrm{k}}\right)$ can be estimated as the slopes of the power-frequency log-log plot of the corresponding PSD in the frequency ranges of concern. Based on the methods described above, a statistical study of the spectral indices is performed at the upstream and downstream regions near the Venusian bow shock in the next section.

\section{Statistical observations}

To statistically differentiate the spectral scaling indices for the upstream and downstream regions near the Venusian bow shock and emphasize the shock geometry effects, we examine the Venus Express magnetic field data for $\sim 7$ years (2006.05-2012.08) and identify the bow shock crossings. Firstly, we select the orbits when the interplanetary magnetic field (IMF) is relatively steady; that is, the directional changes of the IMF between inbound and outbound crossings of bow shock ( 15-min time interval) are less than $30^{\circ}$. Secondly, the shape of the Venusian bow shock can be estimated based on the positions of these two bow shock crossings and the conic section equation $R=L /(1+e \cos \theta)$ with a focus at $\left(x_{0}, 0,0\right)$ and a fixed $e$ of 1.03 during solar minimum (2006-2010) or 1.095 during solar maximum (2011-2012) as reported by Shan et al. (2015), where $R$ is the bow shock distance from the conic focus, $L$ is the conic section semi-latus rectum, and $e$ is the eccentricity. At last, we find 209 orbits/418 shock crossings with steady IMF and welldetermined bow shock model.

We calculate the PSDs of the upstream and downstream intervals of these shock crossings, and then the values of $\alpha_{\mathrm{m}}$ and $\alpha_{\mathrm{k}}$ can be estimated in the corresponding frequency ranges, with the method described above. Figure 2 shows the histograms of $\alpha$ for the upstream and the downstream intervals of the 418 shock crossings in the MHD and kinetic regimes. We find that, as shown in Fig. $2 \mathrm{a}, \mathrm{b}$, the median values of $\alpha_{\mathrm{m}}$ are 1.26 and 1.03 and the mean values are 1.25 and 1.09 with the standard deviations of 1.07 and 1.10 for the upstream and the downstream intervals, respectively. The statistical distributions indicate that these magnetic fluctuations are in a developing and mixed state. From upstream to downstream, the values of $\alpha_{\mathrm{m}}$ show a decreasing trend and the downstream fluctuations tend toward $1 / f$ noise in the MHD regime. This can be ascribed to the fact that collisionless shock physics is mediated by particles and that the injection scale tends to be close to the proton kinetic scale. Since the analysis is performed close to the bow 

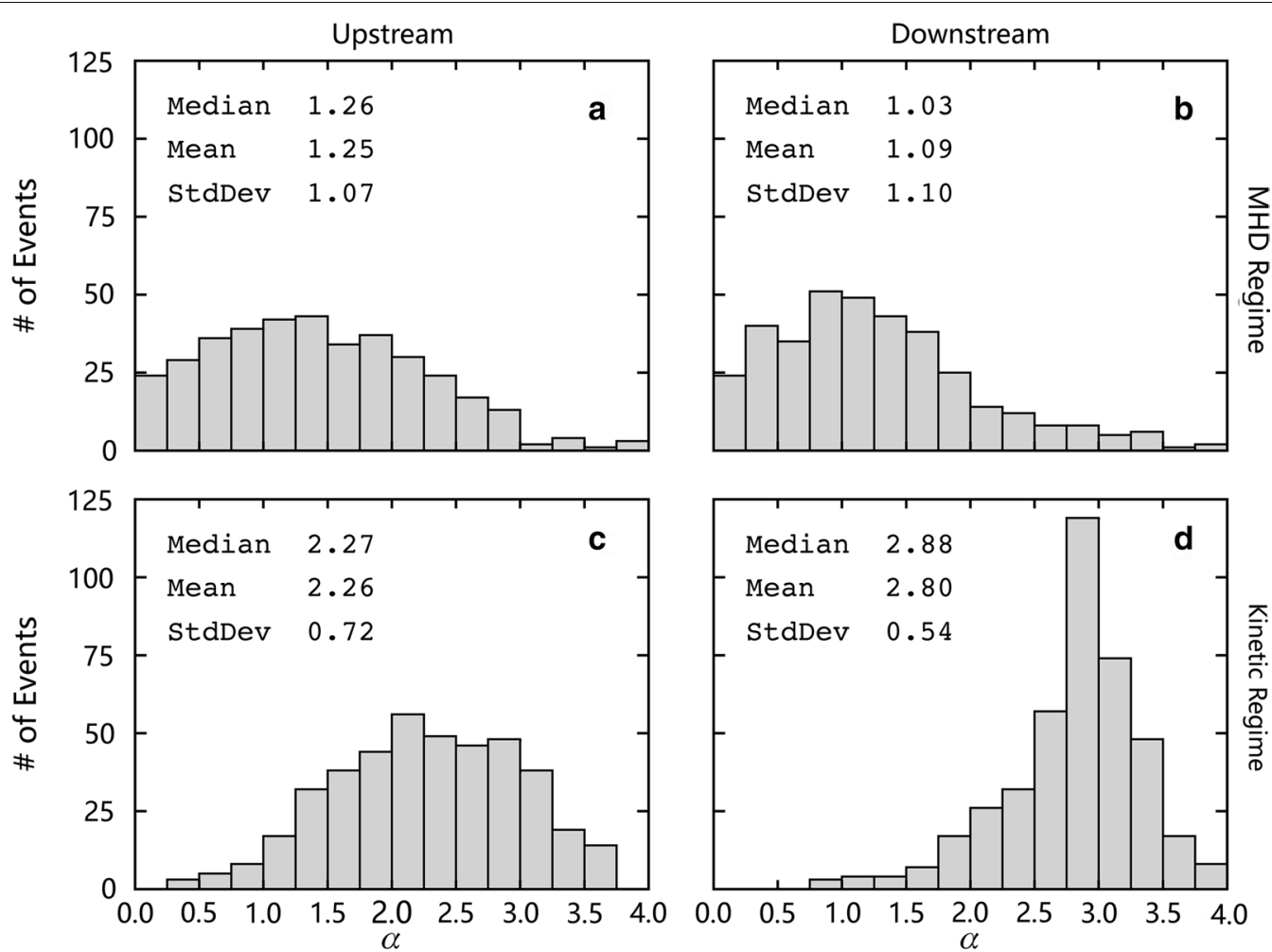

Fig. 2 Histograms of the spectral indices for $\mathbf{a}, \mathbf{c}$ the upstream and $\mathbf{b}$, $\mathbf{d}$ the downstream intervals of the bow shock crossings. $\mathbf{a}, \mathbf{b}$ The histograms for the MHD regime. $\mathbf{c}, \mathbf{d}$ The histograms for the kinetic regime

shock, the turbulence has not fully developed yet. Similar features can also be observed downstream of the Earth bow shock (e.g., Yordanova et al. 2008). Figure 2c, d indicates the median values of $\alpha_{\mathrm{k}}$ are 2.27 and 2.88 and the mean values are 2.26 and 2.80 with the standard deviations of 0.72 and 0.54 for the upstream and the downstream intervals, respectively. The values of $\alpha_{\mathrm{k}}$ for the downstream tend to be larger and concentrated, and the downstream distribution indicates that the kinetic turbulence dominates behind the bow shock. As reported by a previous study (Xiao et al. 2020b), well-developed turbulence is a common phenomenon in the pristine SW near Venus. However, Fig. 2 shows that although some spectral indices indicating the turbulence can still be found, these upstream intervals are rarely turbulence dominant. We infer that the SW fluctuations have been modified before they reach the bow shock.

To be noticed, we can find that the histograms in Fig. 2 exhibit large dispersions. That indicates the complex and diverse magnetic fluctuations near the Venusian bow shock, and the spectral scalings of the fluctuations could be affected by multiple factors. Some previous studies suggested that the bow shock geometry could affect the fluctuations and turbulence in the near-Venusian space (e.g., Luhmann et al. 1983; Xiao et al. 2018). To further investigate the bow shock modifications to the SW fluctuations and turbulence, we split the bow shock crossings into two categories of $Q_{\|}\left(\theta_{\mathrm{BN}}<45^{\circ}\right)$ and $Q_{\perp}\left(\theta_{\mathrm{BN}}>45^{\circ}\right)$ geometries. The shock normal angle $\theta_{\mathrm{BN}}$ can be calculated by the average upstream magnetic field and the estimated local bow shock normal. The average upstream magnetic field is obtained in the 256-s upstream interval of each shock crossing event. The shock normal is determined by the estimated bow shock model with the method described above. Consequently, $115 Q_{\|}$events and $303 Q_{\perp}$ events are obtained, and then we can examine the shock geometry effects on the spectral scalings of fluctuations near the bow shock. Here we show two examples of the magnetic field observations in Fig. 3 for the $Q_{\|}$and the $Q_{\perp}$ bow shock crossings. Figure 3a, b presents the total magnetic field, and Fig. 3c, d presents the magnetic fields in the aberrated VSO coordinate system. Figure $3 \mathrm{a}$, c presents a $Q_{\|}$bow shock $\left(\theta_{\mathrm{BN}} \sim 28.4^{\circ}\right)$ crossing event on May 16, 2006. Figure 3b, $d$ presents a $Q_{\perp}$ bow shock $\left(\theta_{\mathrm{BN}} \sim 84.0^{\circ}\right)$ crossing event on Nov. 18, 2006. This indicates the high level of excited fluctuations upstream of the $Q_{\|}$shock by backstreaming ions in comparison with the $Q_{\perp}$ configuration.

Figure 4 shows the histograms of $\alpha$ for the upstream and the downstream intervals of the $Q_{\|}$(red) and $Q_{\perp}$ 

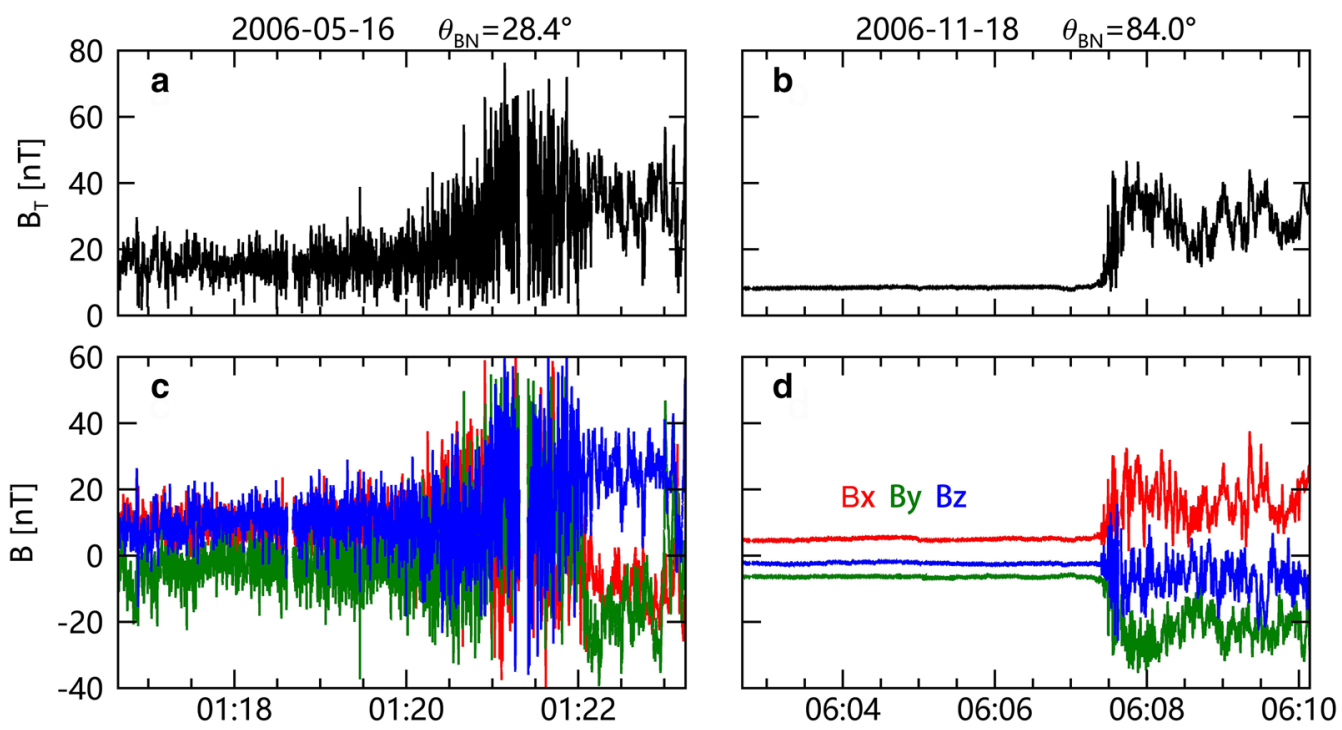

Fig. 3 The time series of the magnetic field magnitude and components for the two types of shock geometry encountered during the Venusian bow shock crossings. a, c The magnetic field observations near a $Q_{\|}$bow shock on 2006 May 16. b. d The magnetic field observations near a $Q_{\perp}$ bow shock on 2006 November 18
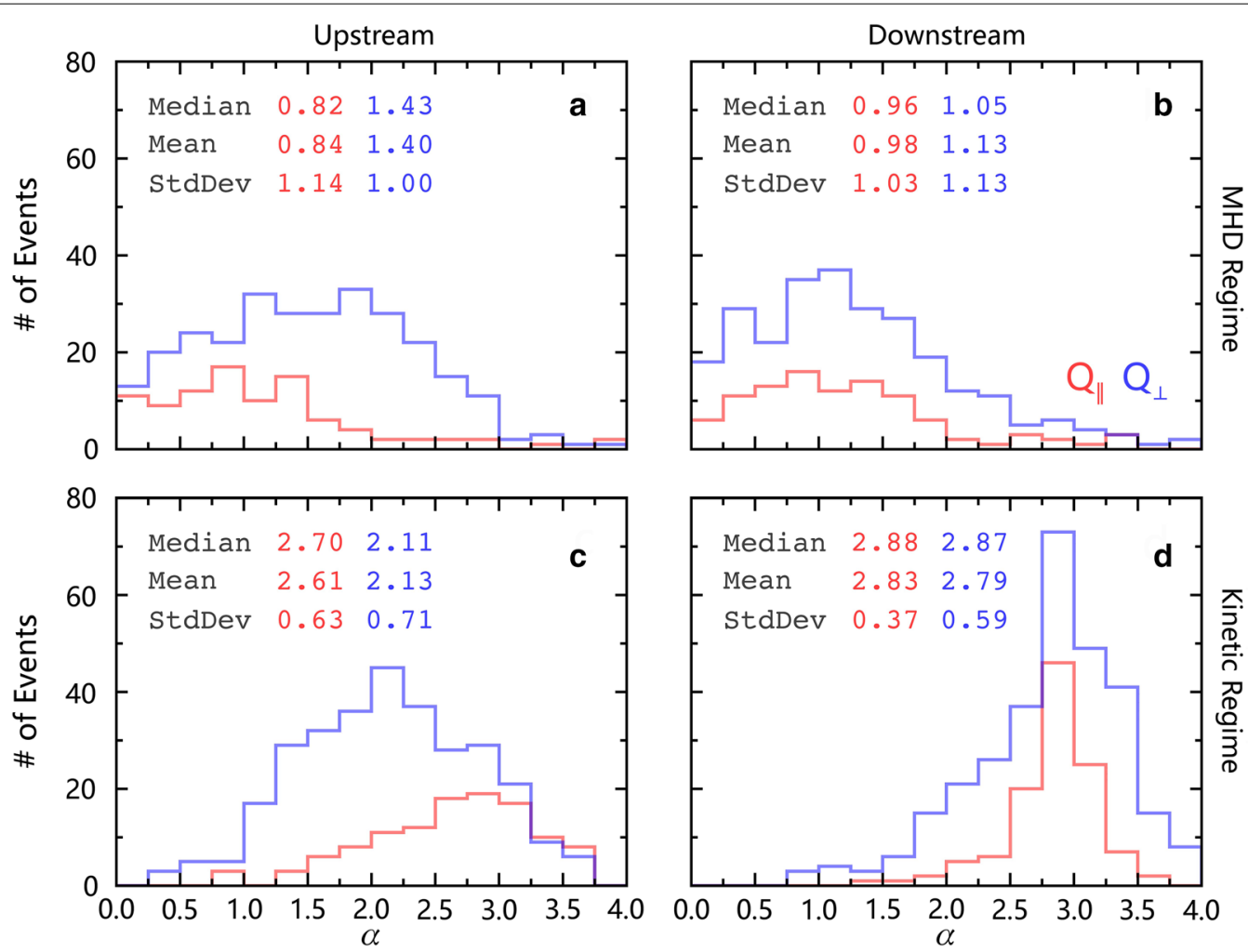

Fig. 4 Histograms of the spectral indices for $\mathbf{a}, \mathbf{c}$ the upstream and $\mathbf{b}$, $\mathbf{d}$ the downstream intervals of the $Q_{\|}$(red) and $Q_{\perp}$ (blue) bow shock crossings. $\mathbf{a}, \mathbf{b}$ The histograms for the MHD regime. $\mathbf{c}, \mathbf{d}$ The histograms for the kinetic regime 
(blue) bow shock crossings. Figure $4 \mathrm{a}$, b shows the histograms of $\alpha_{\mathrm{m}}$. The median values of $\alpha_{\mathrm{m}}$ for the downstream intervals of $Q_{\|}$and $Q_{\perp}$ bow shocks are 0.96 and 1.05, respectively. The similar distributions suggest that, in the MHD regime, the effects of the bow shock on the SW fluctuations and turbulence are independent on the shock geometry. However, the values of $\alpha_{\mathrm{m}}$ for the upstream intervals are obviously related to the shock geometry. For the upstream intervals of $Q_{\|}$and $Q_{\perp}$ bow shocks, the median values of $\alpha_{\mathrm{m}}$ are 0.82 and 1.43, respectively. Both distributions upstream and downstream of the $Q_{\|}$bow shocks present two peaks at $\sim 1$ and $\sim 1.5$. The spectral scalings do not show significant differences between the fluctuations upstream and downstream of the $Q_{\|}$shocks. The MHD fluctuations upstream of the $Q_{\perp}$ bow shocks are also in a mixed state that some fluctuations might be modified or still in developing, but the distribution is more like the SW with pre-existing turbulence. Obvious differences exist between the upstream and downstream intervals of the $Q_{\perp}$ shocks, which could result from the waves excited behind the $Q_{\perp}$ shocks. We infer that the pre-existing MHD turbulence from the SW could be more prone to reaching the $Q_{\perp}$ bow shocks but start to be modified before reaching the $Q_{\|}$bow shocks. The modification might be due to the waves generated near the bow shock.

Figure 4c, d shows the histograms of $\alpha_{\mathrm{k}}$. The median values of $\alpha_{\mathrm{k}}$ are 2.88 and 2.87 for the downstream intervals of $Q_{\|}$and $Q_{\perp}$ bow shocks, respectively. The distributions indicate that the kinetic turbulence is significantly dominant for the downstream intervals, and it is not shock geometry dependent. The upstream kinetic fluctuations also show a difference between the $Q_{\|}$and $Q_{\perp}$ bow shock crossings. The median values of $\alpha_{\mathrm{k}}$ for the upstream intervals of $Q_{\|}$and $Q_{\perp}$ bow shocks are, respectively, 2.70 and 2.11 . The statistical kinetic spectral scalings of the fluctuations near the $Q_{\|}$bow shocks do not change significantly as near the $Q_{\perp}$ bow shocks, but the values of $\alpha_{\mathrm{k}}$ for the downstream intervals have a much more concentrated distribution. This indicates that the downstream kinetic turbulence is mainly developed behind the bow shock but not penetrate from the upstream. The kinetic turbulence can also be developed upstream of the $Q_{\|}$bow shocks. We infer that the SW kinetic fluctuations could be affected by the Venusian bow shock at the upstream region and the upstream difference of the $Q_{\|}$and $Q_{\perp}$ bow shock might result from the more backstreaming ions upstream of the $Q_{\|}$bow shock.

Based on the results from Fig. 4, we find the shock geometry does influence the spectral scalings of magnetic fluctuations upstream and downstream of the Venusian bow shock. However, a large dispersion is still shown in some histograms. Therefore, the shock geometry is one key factor but not the only factor in the propagation of the fluctuations across the Venusian bow shock.

\section{Discussion and conclusions}

In this paper, we use the magnetic field data of Venus Express from 2006.05 to 2012.08 to investigate the spectral scalings variations of the magnetic field fluctuations near the Venusian bow shock. The spectral indices are calculated in the MHD and kinetic regimes for the upstream and downstream intervals close to the Venusian bow shock. There are $115 Q_{\|}$and $303 Q_{\perp}$ shock crossings selected in this study. Based on the statistical results, the shock effects on the spectral scalings near the Venusian bow shocks are examined.

We find the Venusian bow shock tends to flatten the spectra of upstream MHD fluctuations and steepen the kinetic spectra. At the downstream regions, the MHD magnetic fluctuations and turbulence tend to be modified to $1 / f$ noise and the kinetic turbulence can be fully developed behind the shock, which is consistent with the previous studies (e.g., Vörös et al. 2008a; Xiao et al. 2020b). This suggests that the energy cascade and dissipation near Venus can be modified by the Venusian bow shock. The spectral indices for the downstream intervals show this shock modification is independent on the shock geometry. However, we find the upstream spectral scalings are associated with the shock geometry. In the MHD regime, the spectra upstream of $Q_{\|}$bow shocks are flatter than that of $Q_{\perp}$ bow shocks. In the kinetic regime, the spectra upstream of $Q_{\|}$bow shocks are steeper than that of $Q_{\perp}$ bow shocks. As reported by a previous study, the $\alpha_{\mathrm{m}}$ in the pristine solar wind far upstream of the Venusian bow shock is near Kolmogorov scaling value and the values of $\alpha_{\mathrm{k}}$ are typically $\sim 2.5-3$ (Xiao et al. 2020b). Our results in this study indicate that the bow shock effects on the SW fluctuations could begin at the upstream region, which might result from reflected ions and newborn pickup ions. For example, the ULF waves excited by backstreaming ions exhibit their frequency range of $0.3-0.5 f_{p}$ (e.g., Shan et al. 2016), which could lead to a decrease of $\alpha_{\mathrm{m}}$, and the whistler-mode waves generated upstream of the bow shock exhibit their frequency range from several $f_{p}$ to $2 \mathrm{~Hz}$ (e.g., Russell 2007), which could lead to an increase of $\alpha_{\mathrm{k}}$. For validating the PSDs in the spacecraft frame, the possibility of violating the Taylor hypothesis need to be considered, especially at high frequencies and in an environment close to a shock (e.g., Klein et al. 2014). Unfortunately, because of the limited observational data, it is hard to detect the violation of the Taylor hypothesis in this study. Multiple wave modes can be generated near the bow shock, and sometimes the spectral shape might not be clear for the fluctuations in the shock upstream and shock downstream 
regions. The values of $\alpha$ in this region exhibit a large variation. To interpret these fluctuations, we further examine their compression and rotation senses (Arthur et al. 1976; Means 1972), as with some prior studies on magnetic field fluctuations near Venus (e.g., Guicking et al. 2010; Du et al. 2010; Xiao et al. 2017). The histograms of these fluctuation properties are shown in Fig. 5 for the $Q_{\|}$(red) and $Q_{\perp}$ (blue) events.

The transverse and compressional ratio $(\zeta)$ of fluctuations is defined as $\left(P_{\perp}-P_{\|}\right) / P_{\mathrm{T}}$, where $P_{\perp}$ is the transverse power of the fluctuations with respect to the ambient magnetic field, $P_{\|}$is the compressional power, and $P_{\mathrm{T}}$ is the total power. The range of $\zeta$ is from -1 to 1. A positive (negative) ratio means the transverse power is higher (lower) than the compressional power, and a ratio of 1 indicates that this fluctuation is purely transverse. Figure $5 \mathrm{a}-\mathrm{d}$ shows the histograms of $\zeta$ in the MHD and kinetic regimes. In the MHD regime, we can find the upstream fluctuations are mainly transverse, while more compressional fluctuations are present downstream, especially behind the $Q_{\perp}$ bow shock. That might be due to the compressional waves generated by the mirror mode instability (e.g., Volwerk et al. 2008). In the kinetic regime, the transverse fluctuations dominate in the region near the Venusian bow shock, and this nature is shock geometry independent.

The ellipticity $(\varepsilon)$ of fluctuations is defined as the ratio of the minor to major axis of the polarization ellipse transcribed by the field variations of the components transverse to the ambient field (Samson and Olson 1980). The sign indicates the direction of rotation of the polarization ellipse, i.e., the rotation sense about the ambient field
(Means 1972). Negative signs refer to left-handed polarized waves and positive signs refer to right-handed polarized waves. The waves of linear and circular polarization correspond to the $\varepsilon$ of 0 and \pm 1 , respectively. Figure $5 \mathrm{e}-\mathrm{h}$ shows the histograms of $\varepsilon$ in the MHD and kinetic regimes. The histograms indicate that many right-handed polarized fluctuations can be generated behind the bow shock, and they could be excited by the pickup ions. In addition, we consider that these left-handed polarized waves in kinetic regime, which are hardly observed downstream, are related to the upstream whistler-mode waves or the so-called $1-\mathrm{Hz}$ waves generated at the bow shock and propagating upstream (e.g., Russell 2007; Xiao et al. 2020c). These waves are elliptically polarized and intrinsically right-handed polarized; however, they are observed as left-handed elliptically polarized in spacecraft frame due to the Doppler shifting effect.

At the $Q_{\|}$shocks, some ions can escape into the foreshock region, and a variety of large-amplitude waves can be excited by these backstreaming ions (e.g., Delva et al. 2011; Collinson et al. 2012; Shan et al. 2014). These waves generated in the foreshock can be convected to the downstream side across the $Q_{\|}$bow shock (e.g., Luhmann et al. 1983; Shan et al. 2014). That might be a reason of the statistical finding that there are no significant variations of the spectral scalings of magnetic fluctuations near the $Q_{\|}$bow shocks. This suggests that the $Q_{\|}$bow shock is an important channel of the energy injection and dissipation in the interaction of the SW with Venus. More fluctuations and higher energies are injected at $Q_{\|}$rather than $Q_{\perp}$ shocks. At the $Q_{\perp}$ shocks, these ions will generally gyrate back and then excite waves behind the shocks

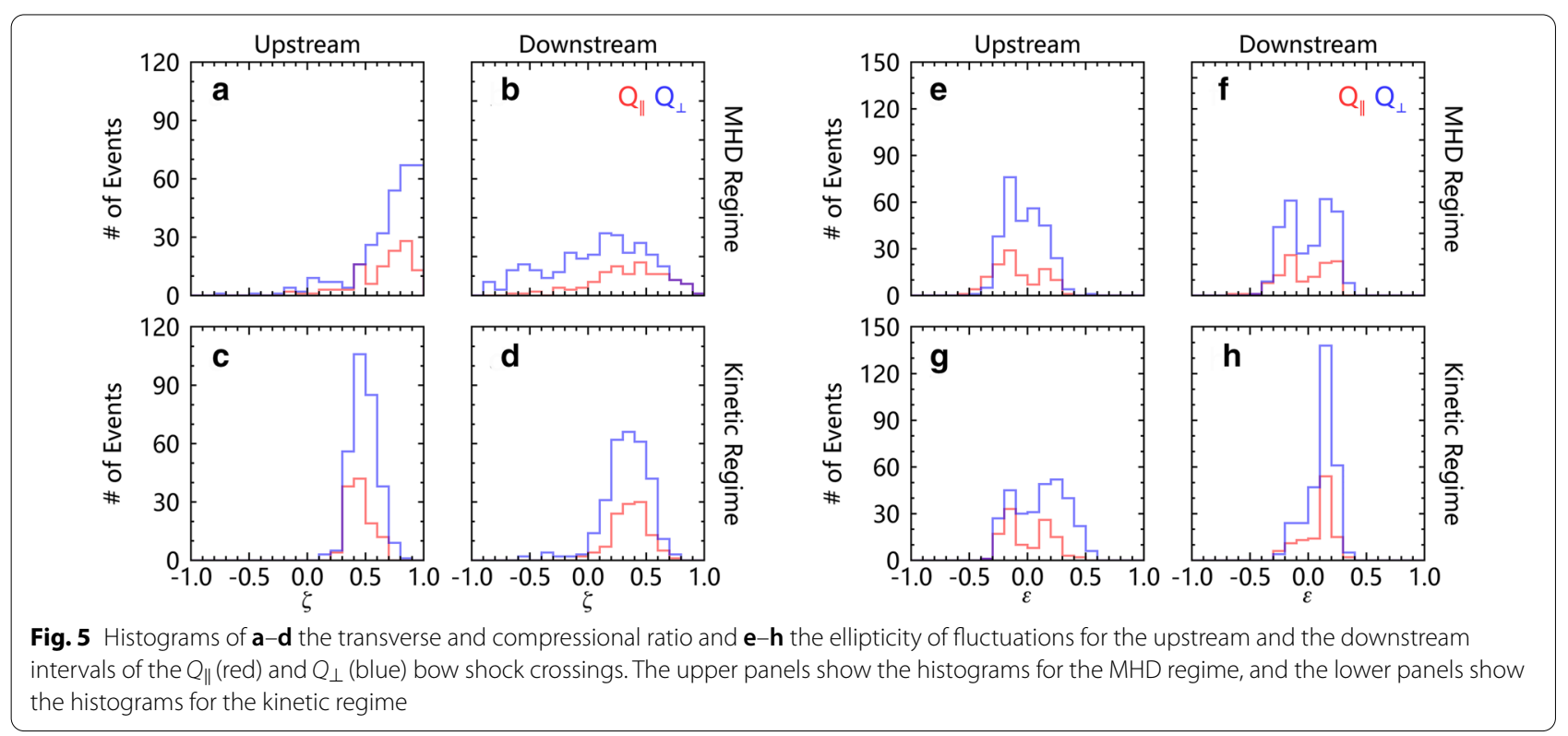


(e.g., Volwerk et al. 2008). These waves could result in the changes of downstream spectral scalings. However, in some cases, the waves upstream of $Q_{\perp}$ shocks can be transmitted into the downstream, and the downstream spectra could also be similar to the upstream except an enhanced amplitude (e.g., Lu et al. 2009). Thereby, the spectral scalings variations across the bow shock could be controlled by multiple factors. In this study, we find the shock geometry is an important factor. Other possible factors affecting the Venusian bow shock modification to the SW fluctuations will be our future topics.

\section{Acknowledgements}

The authors are grateful to the supports of NSFC (41904156, 41974205 $41774171,41774167,41804157)$, China Postdoctoral Science Foundation (2019M651271), and the pre-research Project on Civil Aerospace Technologies (No. D020103) funded by CNSA. The authors also acknowledge the financial support of Shenzhen Science and Technology Research Program (JCYJ20180306171918617) and Shenzhen Science and Technology Program (Group No. KQTD20180410161218820), and the support of CAS Center for Excellence in Comparative Planetology.

\section{Authors' contributions}

SDX initiated the investigation and prepared the original manuscript. TLZ supervised the investigation. MYW and GQW participated in the discussions and reviewed the manuscript. YQC and TLZ gave the suggestions of the manuscript. All authors read and approved the final manuscript.

\section{Funding}

This work was supported by NSFC grants $(41904156,41974205,41774171$, 41774167 and 41804157), the project funded by China Postdoctoral Science Foundation (2019M651271), the pre-research Project on Civil Aerospace Technologies (No. D020103) funded by CNSA, Shenzhen Science and Technology Research Program (JCYJ20180306171918617), and Shenzhen Science and Technology Program (Group No. KQTD20180410161218820). Tielong Zhang was supported by CAS Center for Excellence in Comparative Planetology.

\section{Availability of data and materials}

Venus Express magnetic field data are available in the ESA's Planetary Science Archive (ftp://psa.esac.esa.int/pub/mirror/VENUS-EXPRESS/).

\section{Consent for publication}

Not applicable.

\section{Competing interests}

The authors declare that they have no competing interests.

\section{Author details}

${ }^{1}$ Harbin Institute of Technology, Shenzhen, China. ${ }^{2}$ CAS Key Laboratory of Geospace Environment, University of Science and Technology of China, Hefei, China. ${ }^{3}$ Space Research Institute, Austrian Academy of Sciences, Graz, Austria.

Received: 5 May 2020 Accepted: 21 December 2020

Published online: 07 January 2021

\section{References}

Alexandrova O, Carbone V, Veltri P, Sorriso-Valvo L (2008) Small-scale energy cascade of the solar wind turbulence. Astrophys J 674(2):1153-1157

Alexandrova O, Saur J, Lacombe C, Mangeney A, Mitchell J, Schwartz SJ et al (2009) Universality of solar-wind turbulent spectrum from mhd to electron scales. Phys Rev Lett 103(16):165003

Arthur CW, McPherron RL, Means JD (1976) A comparative study of three techniques for using the spectral matrix in wave analysis. Radio Sci 11(10):833-845. https://doi.org/10.1029/RS011i010p00833
Bruno R, Carbone V (2013) The solar wind as a turbulence laboratory. Living Rev Sol Phys 10(1):1-208

Chen CHK, Leung L, Boldyrev S, Maruca BA, Bale SD (2014) Ion-scale spectral break of solar wind turbulence at high and low beta. Geophys Res Lett 41:8081-8088. https://doi.org/10.1002/2014GL062009

Collinson GA, Wilson LB, Sibeck DG, Shane N, Zhang TL, Moore TE, Coates AJ, Barabash S (2012) Short large-amplitude magnetic structures (SLAMS) at Venus. J Geophys Res 117:A10221. https://doi.org/10.1029/2012JA017838

Delva M, Mazelle C, Bertucci C, Volwerk M, Vörös Z, Zhang TL (2011) Proton cyclotron wave generation mechanisms upstream of Venus. J Geophys Res 116:A02318. https://doi.org/10.1029/2010JA015826

Du J, Zhang TL, Baumjohann W, Wang C, Volwerk M, Vörös Z, Guicking L (2010) Statistical study of low-frequency magnetic field fluctuations near Venus under the different interplanetary magnetic field orientations. J Geophys Res 115:A12251. https://doi.org/10.1029/2010JA015549

Dwivedi NK, Schmid D, Narita Y, Kovács P, Vörös Z, Delva M, Zhang T (2015) Statistical investigation on the power-law behavior of magnetic fluctuations in the Venusian magnetosheath. Earth Planets Space 67(1):137

Guicking L, Glassmeier KH, Auster HU, Delva M, Motschmann U, Narita Y, Zhang TL (2010) Low-frequency magnetic field fluctuations in Venus' SW interaction region: Venus Express observations. Ann Geophys 28:951-967

Kiyani KH, Chapman SC, Khotyaintsev YV, Dunlop MW, Sahraoui F (2009) Global scale-invariant dissipation in collisionless plasma turbulence. Phys Rev Lett 103(7):075006

Klein K, Howes G, Tenbarge J (2014) The violation of the Taylor hypothesis in measurements of solar wind, turbulence. Astrophys J Lett 790(2):L20

Lu Q, Hu Q, Zank GP (2009) The interaction of Alfvén waves with perpendicular shocks. Astrophys J 706(1):687

Luhmann JG (1986) The SW interaction with Venus. Space Sci Rev 44:241-306

Luhmann JG, Tatrallyay M, Russell CT, Winterhalter D (1983) Magnetic field fluctuations in the Venus magnetosheath. Geophys Res Lett 10(8):655-658. https://doi.org/10.1029/GL010i008p00655

Means JD (1972) Use of the three-dimensional covariance matrix in analyzing the polarization properties of plane waves. J Geophys Res 77(28):55515559. https://doi.org/10.1029/JA077i028p05551

Phillips JL, McComas DJ (1991) The magnetosheath and magnetotail of Venus. Space Sci Rev 55:1-80. https://doi.org/10.1007/BF00177135

Rong ZJ, Barabash S, Futaana Y, Stenberg G, Zhang TL, Wan WX, Wei Y, Wang X-D, Chai LH, Zhong J (2014) Morphology of magnetic field in near-Venus magnetotail: Venus Express observations. J Geophys Res Space Phys 119:8838-8847. https://doi.org/10.1002/2014JA020461

Ruhunusiri S, Halekas JS, Espley JR, Mazelle C, Brain D, Harada Y, DiBraccio GA, Livi R, Larson DE et al (2017) Characterization of turbulence in the Mars plasma environment with MAVEN observations. J Geophys Res Space Phys 122(1):656-674. https://doi.org/10.1002/2016JA023456

Russell CT (2007) Upstream whistler-mode waves at planetary bow shocks: a brief review. J Atmos Sol Terr Phys 69(14):1739-1746

Samson JC, Olson JV (1980) Some comments on the descriptions of the polarization states of waves. Geophys J Roy Astron Soc 61:115-129. https://doi. org/10.1111/j.1365-246X.1980.tb04308.x

Shan L, Lu Q, Wu M, Gao X, Huang C, Zhang T, Wang S (2014) Transmission of large-amplitude ULF waves through a quasi-parallel shock at Venus. J Geophys Res Space Phys 119:237-245. https://doi.org/10.1002/2013」 A019396

Shan L, Lu Q, Mazelle C, Huang C, Zhang T, Wu M et al (2015) The shape of the Venusian bow shock at solar minimum and maximum: revisit based on vex observations. Planet Space Sci 109-110:32-37. https://doi. org/10.1016/j.pss.2015.01.004

Shan L, Mazelle C, Meziane K, Delva M, Lu Q, Ge YS, Du A, Zhang T (2016) Characteristics of quasi-monochromatic ULF waves in the Venusian foreshock. J Geophys Res Space Phys 121:7385-7397. https://doi.org/10.1002/2016J A022876

Slavin JA, Elphic RC, Russell CT, Intriligator DS, Wolfe JH (1979) Position and shape of the Venus bow shock: Pioneer Venus Orbiter observations. Geophys Res Lett 6(11):901-904. https://doi.org/10.1029/GL006i011p00901

Svedhem H, Titov DV, McCoy D, Lebreton JP, Barabash S, Bertaux JL et al (2007) The first European mission to Venus. Planet Space Sci 55(12):1636-1652. https://doi.org/10.1016/j.pss.2007.01.013

Titov DV, Svedhem H, Koschny D, Hoofs R, Barabash S, Bertaux JL et al (2006) Venus Express science planning. Planet Space Sci 54(13-14):1279-1297. https://doi.org/10.1016/j.pss.2006.04.017 
Torrence C, Compo GP (1998) A practical guide to wavelet analysis. Bull Amer Meteor Soc 79:61-78

Volwerk M, Zhang TL, Delva M, Vörös Z, Baumjohann W, Glassmeier K-H (2008) First identification of mirror mode waves in Venus' magnetosheath? Geophys Res Lett 35:L12204. https://doi.org/10.1029/2008GL033621

Vörös Z (2011) Magnetic reconnection associated fluctuations in the deep magnetotail: ARTEMIS results. Nonlinear Process Geophys 18:861-869. https://doi.org/10.5194/npg-18-861-2011

Vörös Z, Baumjohann W, Nakamura R, Volwerk M, Runov A, Zhang TL et al (2004) Magnetic turbulence in the plasma sheet. J Geophys Res 109:A11215. https://doi.org/10.1029/2004JA010404

Vörös Z, Baumjohann W, Nakamura R, Runov A, Volwerk M, Asano Y, Jankovičová D, Lucek EA, Rème H (2007) Spectral scaling in the turbulent Earth's plasma sheet revisited. Nonlinear Process Geophys 14:535-541. https://doi.org/10.5194/npg-14-535-2007

Vörös Z, Zhang TL, Leubner MP, Volwerk M, Delva M, Baumjohann W, Kudela K (2008a) Magnetic fluctuations and turbulence in the Venus magnetosheath and wake. Geophys Res Lett 35:L11102. https://doi. org/10.1029/2008GL033879

Vörös Z, Zhang TL, Leubner MP, Volwerk M, Delva M, Baumjohann W (2008b) Intermittent turbulence, noisy fluctuations, and wavy structures in the Venusian magnetosheath and wake. J Geophys Res 113:E00B21. https:// doi.org/10.1029/2008JE003159

Xiao SD, Zhang TL (2018) Solar cycle variation of the Venus magnetic barrier. Planet Space Sci 158:53-62. https://doi.org/10.1016/j.pss.2018.05.006

Xiao SD, Zhang TL, Baumjohann W (2016) Hemispheric asymmetry in the nearVenusian magnetotail during solar maximum. J Geophys Res Space Phys 121(5):4542-4547. https://doi.org/10.1002/2015JA022093

Xiao SD, Zhang TL, Wang GQ (2017) Statistical study of low-frequency magnetic field fluctuations near Venus during the solar cycle. J Geophys Res Space Phys 122:8409-8418. https://doi.org/10.1002/2017JA023878

Xiao SD, Zhang TL, Vörös Z (2018) Magnetic fluctuations and turbulence in the Venusian magnetosheath downstream of different types of bow shock. J
Geophys Res Space Phys 123:8219-8226. https://doi.org/10.1029/2018J A025250

Xiao SD, Zhang TL, Vörös Z, Wu MY, Wang GQ, Chen YQ (2020a) Turbulence near the Venusian bow shock: Venus Express observations. J Geophys Res Space Phys. https://doi.org/10.1029/2019JA027190

Xiao SD, Wu MY, Wang GQ, Wang G, Chen YQ, Zhang TL (2020b) Turbulence in the near-Venusian space: Venus Express observations. Earth Planet Phys 4(1):82-87. https://doi.org/10.26464/epp2020012

Xiao SD, Wu MY, Wang GQ, Chen YQ, Zhang TL (2020c) Survey of 1-Hz waves in the near-Venusian space: Venus Express observations. Planet Space Sci 187:104933. https://doi.org/10.1016/j.pss.2020.104933

Yordanova E, Vaivads A, Andre M, Buchert SC, Voeroes Z (2008) Magnetosheath plasma turbulence and its spatiotemporal evolution as observed by the Cluster spacecraft. Phys Rev Lett 100(20):205003

Zhang TL, Luhmann JG, Russell CT (1991) The magnetic barrier at Venus. J Geophys Res 96:11145-11153

Zhang TL, Baumjohann W, Delva M, Auster H-U, Balogh A, Russell CT et al (2006) Magnetic field investigation of the Venus plasma environment: expected new results from Venus Express. Planet Space Sci 54(1314):1336-1343. https://doi.org/10.1016/j.pss.2006.04.018

Zhang TL et al (2008a) Induced magnetosphere and its outer boundary at Venus. J Geophys Res 113:E00B20. https://doi.org/10.1029/2008JE003215

Zhang TL, Delva M, Baumjohann W et al (2008b) Initial Venus Express magnetic field observations of the magnetic barrier at solar minimum. Planet Space Sci 56(6):790-795

Zhang TL et al (2008c) Initial Venus Express magnetic field observations of the Venus bow shock location at solar minimum. Planet Space Sci 56:785-789. https://doi.org/10.1016/j.pss.2007.09.012

\section{Publisher's Note}

Springer Nature remains neutral with regard to jurisdictional claims in published maps and institutional affiliations.

\section{Submit your manuscript to a SpringerOpen ${ }^{\circ}$ journal and benefit from:}

- Convenient online submission

- Rigorous peer review

- Open access: articles freely available online

- High visibility within the field

- Retaining the copyright to your article

Submit your next manuscript at $\boldsymbol{\nabla}$ springeropen.com 